\title{
Hyperbolic inverse mean curvature flow with forced term: evolution of plane curves
}

\author{
Ran Ding ${ }^{1}$ and Zenggui Wang ${ }^{1}$ \\ ${ }^{1}$ Liao Cheng University
}

February 27, 2022

\begin{abstract}
The motion of plane curves specified by hyperbolic inverse mean curvature with a constant force term is considered. We proved that this flow remains the convexity for any forced term. Furthermore, we give an example to understand how the constant forced term $\$ c \$$ affects this hyperbolic inverse mean flow. Particularly, the asymptotic behavior of the flow under different initial conditions is discussed.
\end{abstract}

\section{Hosted file}

Manuscript.pdf available at https://authorea.com/users/462518/articles/557922-hyperbolicinverse-mean-curvature-flow-with-forced-term-evolution-of-plane-curves 